\title{
ATF4 regulates neuronal death in models of Parkinson's Disease
}

3 Matthew D. Demmings ${ }^{2,3,4}$, Gillian N. Petroff ${ }^{1,3,4}$, Heather E. Tarnowski-Garner ${ }^{3,4}$,

4 Sean P. Cregan ${ }^{\dagger 1,3,4}$

5 Department of Physiology and Pharmacology ${ }^{1}$, Neuroscience Program², Robarts Research

6 Institute 3 , University of Western Ontario ${ }^{4}$

8 Correspondence should be addressed to Sean P. Cregan, Robarts Research Institute,

9 University of Western Ontario, 1151 Richmond Street, London, ON, Canada, N6A 3K7, E-mail:

10 scregan@robarts.ca

11

12

\section{Abstract}

14 Parkinson's Disease (PD) is characterized by the loss of dopaminergic neurons in the substantia

15 nigra resulting in severe motor impairments. However, the mechanisms underlying this neuronal

16 loss remain largely unknown. Activating Transcription Factor 4 (ATF4), a key mediator of the

17 Integrated Stress Response (ISR), is a transcription factor that during prolonged activation can

18 induce the expression of pro-apoptotic target genes. Oxidative stress and ER stress have been

19 implicated in PD and these factors are known to activate the ISR. In this study, we have

20 determined, that both PD neurotoxins (MPP+ and 6-OHDA) and $\alpha$-synuclein aggregation induced

21 by pre-formed human alpha-synuclein fibrils (PFFs) cause sustained upregulation of ATF4

22 expression in mouse primary cortical and mesencephalic neurons. Furthermore, we demonstrate

23 that PD neurotoxins induce the expression of the pro-apoptotic factors Chop, Trb3 and Puma in

24 an ATF4-dependent manner. Importantly, using neurons derived from ATF4 +/+ and ATF4 -/-

25 mice, we demonstrate that ATF4 promotes neuronal apoptosis and dopaminergic cell loss in

26 cellular models of PD. Finally, we demonstrate that the elF2 $\alpha$ kinase inhibitor C16 suppresses

$27 \mathrm{MPP}+$ and 6-OHDA induced ATF4 activation and protects against PD neurotoxin induced

28 neuronal death. Taken together these results indicate that ATF4 is a key regulator of 
dopaminergic cell death induced by PD neurotoxins and pathogenic $\alpha$-synuclein aggregates and highlight the ISR as a potential therapeutic target in PD.

\section{Introduction}

35 Parkinson's disease (PD) is the second most common neurodegenerative disorder and is

36 characterized by distinct motor symptoms including bradykinesia, postural rigidity and resting

37 tremor (Postuma et al., 2015). The motor impairments in PD result from the progressive loss of

38 dopaminergic neurons in the substantia nigra pars compacta and the associated depletion of

39 striatal dopamine (Damier, Hirsch, Agid, \& Graybiel, 1999). A hallmark of PD is the appearance of

40 intracellular protein inclusions called Lewy Bodies in which aggregates of misfolded $\alpha$-synuclein

41 are a major component (Spillantini et al., 1997). Although the majority of PD cases are sporadic,

42 risk genes including PARK1 ( $\alpha$-synuclein), PARKIN, PINK1, DJ-1 and LRRK2, have been identified in

43 familial forms of the disease (reviewed by Zeng, Geng, Jia, Chen, \& Zhang, 2018). Unfortunately,

44 there is no cure for PD and while current treatments temporarily ameliorate some of the clinical

45 symptoms, they do not mitigate the underlying degenerative processes.

47 Numerous studies implicate oxidative stress and ER stress as major factors contributing to 48 dopaminergic neuronal cell death in PD. Indeed, markers of oxidative damage including DNA

49 damage, lipid peroxidation and aggregates of oxidized proteins have been reported in post-

50 mortem brain tissue of PD patients (Baba et al., 1998; Bosco et al., 2006). The role of oxidative

51 stress is also supported by the finding that environmental toxins (paraquat, rotenone) and

52 neurotoxic agents (MPTP, 6-OHDA) can inhibit mitochondrial function and increase the

53 production of reactive oxygen species cause loss of dopaminergic neurons in rodents, non-

54 human primates and humans (Bove, Prou, Perier, \& Przedborski, 2005). Furthermore, several

55 familial-linked PD genes including Parkin, Pink1 and DJ-1 have been implicated in the regulation

56 of mitochondrial function, mitophagy and ROS production (Clark et al., 2006; Geisler et al., 2010;

57 Lee, Nagano, Taylor, Lim, \& Yao, 2010; Narendra et al., 2010). In addition to oxidative stress, 
deficits in cellular proteostasis pathways associated with the accumulation and aggregation of misfolded proteins such as $\alpha$-synuclein is thought to contribute to neurodegeneration in PD

60 (Kaushik \& Cuervo, 2015). One component of the proteostasis network that is affected in PD is

61 the folding capacity of the endoplasmic reticulum (ER) resulting in ER stress and activation of the

62 unfolded protein response (UPR) (Mercado, Castillo, Soto, \& Sidhu, 2016; Michel, Hirsch, \&

63 Hunot, 2016). Indeed, markers of ER stress are commonly observed in post-mortem brain tissue

64 derived from PD patients as well as in a variety of cellular and animal models of PD (Colla et al.,

65 2012; Heman-Ackah et al., 2017; Holtz \& O'Malley, 2003; Hoozemans et al., 2007; Mercado et

66 al., 2018b; Ryu et al., 2002; Slodzinski et al., 2009).

68 The Integrated Stress Response (ISR) is a cell signaling pathway that is activated in response to 69 diverse stress stimuli including oxidative stress and ER stress (Pakos-Zebrucka et al., 2016). Four

70 kinases have been implicated in the initiation of the ISR including PERK (PKR-like ER kinase), PKR

71 (protein kinase double stranded RNA-dependent), GCN2 (general control non-depressible-2),

72 and HRI (heme-regulated inhibitor) (Donnelly, Gorman, Gupta, \& Samali, 2013). The ISR kinases

73 are activated in a stress specific manner and phosphorylate the translation initiation factor elF $2 \alpha$

74 (Lu et al., 2004). Phosphorylation of elF2 $\alpha$ results in attenuation of general protein translation

75 but permits the selective translation of stress-induced mRNAs including that of Activating

76 Transcription Factor 4 (ATF4) a member of the ATF/CREB family of basic-region leucine zipper

77 transcription factors (Singleton \& Harris, 2012). ATF4 regulates the expression of genes involved

78 in amino acid metabolism and redox homeostasis that promote cell recovery (Harding et al.,

79 2003). However, upon sustained activation ATF4 can also induce the expression of factors that

80 have been implicated in promoting cell death including CHOP and TRB3 (Fawcett, Martindale,

81 Guyton, Hai, \& Holbrook, 1999; Ohoka, Yoshii, Hattori, Onozaki, \& Hayashi, 2005; Zinszner et al.,

82 1998). Induction of ATF4 has been reported in cellular and animal models of PD as well as in

83 post-mortem brain tissue of PD patients (Mercado et al., 2018a; Ryu et al., 2002; Sun et al.,

84 2013). However, the role of ATF4 in regulating neuronal survival remains controversial.

85 Therefore, in the present study we investigated the role of ATF4 in primary neurons exposed to 86 PD neurotoxins (MPP+ and 6-OHDA) and $\alpha$-synuclein preformed fibrils. 
ATF4 in PD Neuronal Death

MD Demmings

\section{Materials and Methods}

93

94 Animals. All animal procedures were performed as per guidelines set by the Animal Care

95 Committee at Western University, in accordance with the Canadian Council on Animal Care.

96 Mice carrying an ATF4-null mutation were obtained from Drs. Tim Townes (University of

97 Alabama, Birmingham, AL). All mice were maintained on a C57BL/6 background. Animals were

98 genotyped as previously described (Masuoka \& Townes, 2002).

99

100 Primary cortical and mesencephalic neuron culture. Cortical neurons were dissociated from

101 embryonic days 14.5-15.5 embryo's and cultured in Neurobasal plus media (ThermoFisher,

102 \#A35829-01) supplemented with B27 plus (ThermoFisher, \#A35828-01), 0.5x Glutamax

103 (ThermoFisher, \#35050-061), and 50U/mL penicillin: $50 \mu \mathrm{g} / \mathrm{mL}$ streptomycin (PenStrep;

104 ThermoFisher, \#15140-122). Mesencephalic neurons were obtained using a protocol modified

105 from Gaven, Marin, and Claeysen, (2014) and cultured in the same media conditions as cortical

106 neurons.

107

108 Drugs. Drug treatments were initiated when neurons were 5-7 DIV. 1-methyl-4-phenylpyridinium

109 iodide (MPP+; Millipore Sigma \#D048), 6-Hydroxydopamine hydrobromide (6-OHDA; Millipore

110 Sigma \#H116), Thapsigargin (TG; Millipore Sigma \#T9033), 6,8-Dihydro-8-(1H-imidazol-5-

111 ylmethylene)-7H-pyrrolo[2,3-g]benzothiazol-7-one [C16] (Tocris \#5382) were prepared in DMSO or

$112 \mathrm{ddH} 20$ and diluted in neuron culture media immediately before administration to cultures at 113 indicated concentrations. 
$115 \alpha$-synuclein preformed fibrils. Human $\alpha$-synuclein monomers (Proteos \#RP-003) were used to

116 generate fibrils based on protocol from Volpicelli-Daley, Luk, \& Lee (2014). Briefly, monomers

117 were shaken at $1000 \mathrm{rpm}\left(37^{\circ} \mathrm{C}\right)$ for 7 days to obtained preformed fibrils, aliquoted and stored at

$118-80^{\circ} \mathrm{C}$. Aliquots were then thawed at room temperature immediately before use. PFFs were

119 diluted in warm medium and sonicated with a probe tip at $10 \%$ power for 30 seconds $(0.5$

120 seconds on, 0.5 seconds off). Sonicated fibrils were added to neuronal culture at a final

121 concentration of $5 \mu \mathrm{g} / \mathrm{mL}$.

122

123 Cell death and survival assays. Neuronal apoptosis was measured by visualizing nuclear

124 morphology in Hoechst 33342 stained cells as described previously (Cregan et al., 2002). Briefly,

125 neurons were fixed using Lana's Fixative (4\% paraformaldehyde, 0.2\% picric acid) for 30 minutes,

126 washed in PBS and stained with Hoechst 33342 (Invitrogen \#H1399) at a concentration of 0.5

$127 \mu \mathrm{g} / \mathrm{mL}$. The fraction of cells exhibiting an apoptotic nuclear morphology characterized by

128 pyknotic and/or fragmented nuclei containing condensed chromatin was scored by an individual

129 blinded to the treatments. Neuronal survival was assessed via Calcein-AM/ Ethidium-Homodimer

130 (Live/Dead) staining (Invitrogen \#L3224). Dye was diluted in warmed neuron medium

131 immediately before use. Calcein-AM $(1 \mu \mathrm{M})$ and Ethidium Homodimer $(3 \mu \mathrm{M})$ was added for 10

132 minutes to determine the ratio of live (Calcein-AM positive) to dead (Ethidium Homodimer

133 positive) cells. In both assays, neurons were visualized by fluorescent microscopy (Olympus) and

134 images were captured using a CCD camera (Q-imaging) and Northern Eclipse software (Empix

135 Imaging).

137 Real-time quantitative PCR (RTqPCR) and quantitative PCR (qPCR). RNA was isolated via Trizol as

138 per guidelines set by manufacturer (Invitrogen \# 15596018) and 40ng was used in one-step Sybr

139 green reverse transcription (RT)-PCR protocol (Qiagen \#204154). RT-PCR was carried out in a CFX

140 Connect Real Time System (Biorad) and changes in gene expression were determined by the

$141 \Delta(\Delta \mathrm{Ct})$ method using S12 transcript for normalization. Values reported for RTqPCR are fold

142 increase in mRNA levels in treated samples relative to paired untreated controls for transcript of

143 interest. RT-PCR amplifications were carried out as follows: $50^{\circ} \mathrm{C}$ for $10 \mathrm{~min}, 95^{\circ} \mathrm{C}$ for $5 \mathrm{~min}, 95^{\circ} \mathrm{C}$ 
144 for $10 \mathrm{sec}$, and $60^{\circ} \mathrm{C}$ for $30 \mathrm{sec}$. Primer sequences used for amplification available upon request.

146 Western blot analysis. To obtain whole-cell lysates, neurons were incubated in lysis buffer (RIPA

147 buffer (Millipore Sigma, \#R0278) supplemented with phosphatase inhibitor cocktail (Millipore

148 Sigma, \#P5726) and protease inhibitor cocktail (Millipore Sigma, \#P8340)) on ice for 30 minutes

149 and soluble extract was recovered via centrifugation. Protein concentrations were determined

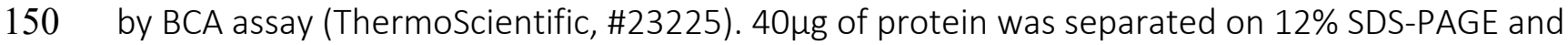

151 transferred to PVDF membrane. Membranes were then blocked for $1 \mathrm{hr}$ at room temperature in

$1525 \%$ Milk prepared in TBST (10 mM Tris, $150 \mathrm{mM} \mathrm{NaCl}, 0.1 \%$ Tween 20). Membranes were the

153 incubated overnight with primary antibodies against ATF4 (1: 5000; Abcam \#184909), Cyclophilin

154 B (1: 5000; Abcam \#178397), and Cleaved Caspase 3 (1: 1000; Cell Signaling Technologies \#9661)

155 at $4^{\circ} \mathrm{C}$. Membranes were then washed in TBST and incubated at room temperature with HRP-

156 conjugated goat anti-rabbit secondary (1: 10000; BioRad \#1706515) for 1 hour. Membranes

157 were then washed again and developed via enhanced chemiluminescence (BioRad \#1705061).

159 Immunocytochemistry. Briefly, neurons were washed with ice cold PBS containing $\mathrm{Na}_{3} \mathrm{VO}_{4}(1 \mathrm{mM}$;

160 Millipore Sigma \#S-6508)) and NaF (25mM; Millipore Sigma \# S-6776). Neurons were then fixed in Lana's

161 fixative (4\% paraformaldehyde, 0.2\% picric acid) and washed in PBS. Cells were then

162 permeabilized in ice cold $100 \% \mathrm{MeOH}$ for 10 mins and washed in PBS. Neurons were then

163 blocked in 2\% BSA for 1 hour and incubated with primary antibodies against MAP2 (1: 500;

164 Abcam \#11267), ATF4 (1:500; Santa Cruz \#SC-200), Cleaved Caspase 3 (1: 400; Cell Signaling

165 Technologies \#9661), Tyrosine Hydroxylase (1:500; Millipore Sigma \#AB152), and p-serine $129 \alpha$ -

166 synuclein (1:500; Abcam 59264) overnight at $4^{\circ} \mathrm{C}$. The next day, cells were washed with PBS and

167 incubated with appropriate AlexFluor (Invitrogen) for 2 hours at room temperature. Neurons

168 were then washed in PBS and Hoechst stained. Coverslips were mounted onto glass microscope

169 slides (VWR \#48311-600) using ProLong Gold mounting glue (Invitrogen \#P36930). Images were

170 obtained using previously described fluorescent microscopy, EVOS Imaging System

171 (ThermoFisher), and confocal images were taken on SP8 microscope (Lieca Microsystems,

172 Germany). 
174 Mitochondrial Superoxide (MitoSox) Staining. 5mM of Mitosox dye (Molecular Probes \#M36008)

175 was prepared as per manufacturer's instructions. Dye was then diluted in warmed neuron

176 medium and added to cultures at final concentration of $200 \mathrm{~nm}$ for 2 hours at $37^{\circ} \mathrm{C} / 5 \% \mathrm{CO}_{2}$.

177 Neurons were then washed with PBS and fixed with Lana's fixative. Subsequently, neurons were

178 incubated with antibodies against MAP2 and appropriate secondary, then mounted and imaged

179 as described above.

180

181 Statistical Analysis. Data are reported as mean \pm standard error of the mean (SEM). Statistical

182 analyses were performed using GraphPad Prism version 8.2 for Mac (GraphPad Software, USA).

183 Statistical significance was considered was set a $\mathrm{p}<0.05$ and determined via t-test or ordinary

184 one-way and two-way ANOVA with Tukey and Sidak post-hoc tests. The ' $n$ ' value indicates the

185 number of independent neuron culture experiments or the number of embryos of each

186 genotype from which neurons were dissociated from.

\section{Results}

191

192 PD neurotoxins MPP+ and 6-OHDA cause sustained activation of ATF4 in neurons

194 The neurotoxins 6-hydroxydopamine (6-OHDA) and 1-methyl-4-phenyl-pyridium (MPP+) cause

195 death of a dopaminergic neurons in vitro and in vivo and have been widely used to investigate

196 cellular mechanisms involved in dopaminergic neuron degeneration in PD (Przedborski \&

197 Ischiropoulos, 2005). These neurotoxins are known to induce mitochondrial dysfunction and to

198 produce ROS that lead to neuronal injury and cell death (Fallon, Matthews, Hyman, \& Beal, 1997;

199 Lotharius, Dugan, \& O’Malley, 1999). Therefore, to investigate the role of ATF4 in PD we initially

200 examined whether these PD-mimetics induce ATF4 expression in primary cortical neurons. As 
shown in Figures $1 \mathrm{~A} \&$ 1B, both MPP+ and 6-OHDA treatments resulted in a robust (>5-fold)

202 increase in ATF4 expression that persisted for at least 16-24 hours.

204 ATF4 is required for transcriptional induction of the pro-apoptotic genes CHOP, Trib3, and PUMA 205 during exposure to PD neurotoxins.

207 ATF4 is a stress responsive transcription factor that can induce the expression of genes involved

208 in amino acid metabolism and redox homeostasis. However, during prolonged stress ATF4 has

209 also been implicated in the regulation of pro-apoptotic genes (Harding 2003; Zinzsner 1998).

210 CHOP, Trib3, and PUMA are known to have pro-apoptotic activity and have been previously

211 reported to be induced by PD neurotoxins and to contribute to subsequent neuronal death

212 (Aimé et al., 2015; Bernstein, Garrison, Zambetti, \& O’Malley, 2011; Bernstein \& O’Malley, 2013;

213 Silva et al., 2005). Therefore, we sought to determine whether the induction of these pro-death

214 factors by PD neurotoxins is regulated by ATF4. To address this, we examined mRNA levels of

215 Chop, Trib3 and Puma in ATF4 +/+ and ATF4 -/- cortical neurons following treatment with PD

216 toxins. Treatment of ATF4+/+ neurons with MPP+ or 6-OHDA resulted in a marked increase in

217 the expression of $\mathrm{CHOP}$, Trib3 and Puma mRNAs (Figure 2A and 2B). However, the increase in

218 expression of these pro-apoptotic factors in response to PD neurotoxins was largely attenuated

219 in ATF4-/- neurons. Taken together these results indicate that sustained activation of ATF4 is

220 required for transcriptional induction of key pro-apoptotic target genes by PD neurotoxins. 
224 Having established that ATF4 promotes the transcriptional induction of several pro-apoptotic

225 target genes following exposure to PD neurotoxins we next investigated whether ATF4 is

226 required for MPP+ and 6-OHDA induced neuronal death. However, since a previous study had

227 indicated that ATF4 can regulate the expression of genes involved in redox homeostasis we

228 wanted to first determine whether the level of oxidative stress induced by PD neurotoxins is

229 affected in ATF4-deficient neurons. To address this, we treated ATF4+/+ and ATF4-/- neurons

230 with MPP+ or 6-OHDA for 8 hours and then assessed the level of mitochondrial superoxide

231 production using MitoSOX Red assay. The MitoSOX probe targets the mitochondria and

232 fluoresces red upon oxidation by superoxide free radicals. As shown in figure 3, both MPP+ and

233 6-OHDA treatments produced significant increases in superoxide levels as determined by the

234 increased MitoSOX Red fluorescence intensity. Furthermore, the level PD-neurotoxin induced

235 superoxide formation was not significantly different between ATF4+/+ and ATF4-/- neurons

236 (Figure 3A and 3B). It was also noted that wildtype and ATF4-deficient neuronal cultures

237 exhibited a similar, healthy neuronal morphology as depicted by MAP2 immunostaining (Figure

238 3B). Next, to determine whether ATF4 is required for PD-neurotoxin induced neuronal apoptosis

239 we treated ATF4+/+ and ATF4-/- neurons with MPP+ or 6-OHDA and then quantified the level of

240 apoptosis as a function of time by assessing nuclear morphology following Hoechst staining. As

241 depicted in Figure 4A, treatment of wild-type neurons with MPP+ or 6-OHDA over a 48-hour

242 period resulted in a substantial increase in the fraction of cells exhibiting an apoptotic

243 morphology characterized by chromatin condensation and/or nuclear fragmentation. In contrast,

244 in ATF4-deficient neurons MPP+ and 6-OHDA treatment resulted in only a modest increase in 
245 apoptosis over this same time frame, and the level of apoptosis was found to be significantly

246 lower than in ATF4+/+ neuronal cultures at both 24 hours and 48 hours post-treatment (Figure

2474 A). Consistent with the apoptotic counts we also found that the level of cleaved (active)

248 Caspase-3 induced by MPP+ and 6-OHDA treatments was markedly reduced in ATF4-/- neurons

249 as compared to ATF4+/+ neurons (Figure 4B and 4C). Next to determine whether ATF4-deficient

250 neurons remained viable following treatment with PD- neurotoxins and were not just dying by a

251 non-apoptotic mode of cell death we assessed neuronal survival in ATF4 +/+ and ATF4 -/- using

252 Calcein-AM/ethidium homodimer staining (Live/Dead assay). As shown in Figure 4D, MPP+ and

253 6-OHDA treatment markedly reduced cell viability in ATF4+/+ neuronal, but not in ATF4-/-

254 neuronal cultures indicating that ATF4-deficient neurons remain viable.

255 Dopaminergic neurons of the substantia nigra are the primary neuronal population of neurons

256 affected in PD. Therefore, we sought to determine whether ATF4 promotes cell death specifically

257 in dopaminergic neurons exposed to PD toxins. To address this question, we generated

258 mesencephalic cultures from the ventral midbrain region of ATF4+/+ and ATF4-/- mouse

259 embryos and subsequently exposed them to MPP+ or 6-OHDA. Only a small portion of the

260 neurons in these mesencephalic cultures are dopaminergic (DA) therefore In order to quantify

261 the extent of DA neuron loss in these models, we quantified the number of neurons with positive

262 immunoreactivity for tyrosine hydroxylase $(\mathrm{TH})$, an enzyme responsible in the conversion of L-

263 tyrosine to L-dihydroxyphenylalanine (L-DOPA) (Nagatsu, Levitt, \& Udenfriend, 1964). As shown

264 in Figure 5, MPP+ treatment resulted in the loss of $63.83 \%$ of the DA neurons in ATF4+/+ cultures

265 as compared to only a 37.05\% reduction in ATF4-/- cultures. Similarly, 6-OHDA treatment

266 resulted in the loss of $52.39 \%$ of the DA neurons in ATF4+/+ cultures as compared to only a 

necessary for PD neurotoxin induced cell death in dopaminergic neurons.

270 Human $\alpha$-synuclein preformed fibrils induces ATF4 dependent neuronal loss.

272 To complement our cellular neurotoxin models, we used human alpha-synuclein to elicit

273 pathogenic aggregates in vitro to model the synucleinopathy present in PD. Previous studies

274 have demonstrated that exogenous preformed $\alpha$-synuclein fibrils ( $\alpha$-Syn PFFs) can be taken up

275 into cultured neurons via endocytosis and induce aggregation of endogenous $\alpha$-synuclein leading

276 to the formation of intracellular inclusions that in turn, can lead to synaptic dysfunction and

277 neuronal death (Volpicelli-Daley et al., 2011). Synuclein incorporated into pathogenic inclusions

278 is highly phosphorylated at Ser129 (Fujiwara et al., 2002). Therefore, to validate this

279 synucleinopathy paradigm we treated cortical neurons with $\alpha$-Syn PFFs and confirmed the

280 formation of phospho-Ser193- $\alpha$-synuclein positive inclusions (Figure 6A). We then performed

281 ATF4 immunostaining to determine whether $\alpha$-Syn PFF induced lesions resulted in the induction

282 of ATF4 expression. Indeed, as shown in Figure 6B, $\alpha$-Syn PFF treated cultures exhibited a

283 significant increase in nuclear ATF4 staining as compared to untreated neurons. We next sought

284 to determined whether $\alpha$-Syn PFF induced inclusions led to neuronal death, and if so whether

285 this was dependent upon ATF4 expression. To address this question, we treated cortical neurons

286 derived from ATF4+/+ and ATF4-/- mice with $\alpha$-Syn PFFs and then assessed apoptosis by

287 examining nuclear morphology. As shown in Figure 6C, $\alpha$-Syn PFF treatment caused a

288 significantly greater induction of apoptosis in ATF4+/+ neurons than in ATF4-/- neurons. Next, we 
289 probed mesencephalic cultures treated with preformed $\alpha$-synuclein fibrils to examine whether

290 loss of ATF4 affects pathogenic $\alpha$-synuclein induced dopaminergic neuron death. A-Syn PFF

291 induced synucleinopathy resulted in the loss of $81.65 \%$ of $\mathrm{TH}+$ dopaminergic neurons in ATF4+/+

292 cultures, but only a 41.85\% reduction in dopaminergic neurons in ATF4-/- cultures (Figure 6D).

293 Taken together these results indicate that ATF4 is activated in response to pathogenic $\alpha$ -

294 synuclein aggregation and promotes neuronal death.

The elF2 $\alpha$ kinase inhibitor C16 inhibits ATF4 activation and protects against neuronal death in

\section{7 cellular models of PD}

299 Oxidative stress and ER stress have been implicated in PD and are known to trigger the

300 Integrated Stress Response and subsequent induction of ATF4 through the activation of elF2 $\alpha$

301 kinases. Given our findings that ATF4 is activated in PD models and promotes neuronal death we

302 reasoned that pharmacological inhibition of elF2 $\alpha$ kinases that contribute to ATF4 activation in

303 response to oxidative stress and ER stress may be neuroprotective in PD. In preliminary

304 experiments we found that the imidazole-oxindole PKR inhibitor commonly known as C16 was

305 able to inhibit ATF4 induction in neurons following treatment with the oxidative stressor arsenite

306 as well as the ER stress inducing agent thapsigargin (data not shown). Therefore, we tested the

307 effects of this elF2 $\alpha$ kinase inhibitor in cellular models of PD. We first investigated whether C16

308 could inhibit PD neurotoxin induced ATF4 activation. As shown in figure 7A, C16 administration

309 significantly reduced the levels of ATF4 induced by both MPP+ and 6-OHDA treatments. We next

310 investigated whether C16 could inhibit the induction of the ATF4-dependent pro-apoptotic 
311 target genes Chop and Trib3. Consistent with the ability of C16 to inhibit ATF4 induction, we

312 found that the transcriptional induction of Chop and Trb3 mRNAs by MPP+ and 6-OHDA was

313 significantly attenuated in the presence of C16 (Figure 7B). Given that C16 decreased ATF4

314 protein and represses transcriptional activation of its downstream targets, we next evaluated

315 whether C16 is neuroprotective in PD neurotoxin paradigms. To test this, we treated cortical

316 neurons with MPP+ or 6-OHDA in the presence or absence of C16 and then assessed the level of

317 apoptotic cell death after 24 hours. As shown in Figure 7C, administration of C16 markedly

318 reduced the level of apoptotic cell death induced by both MPP+ $(63.24$ vs $34.94 \%, p<0.05)$ and 6-

$319 \operatorname{OHDA}(71.34 \%$ vs $45.81 \%, \mathrm{p}<0.05)$.

327 Parkinson's disease is characterized by the loss of dopaminergic neurons, leading to a reduction

328 of dopamine levels, which produce the cardinal motor symptoms of the disease. Currently,

329 treatments are directed towards dopamine replacement therapies, which provide some

330 symptomatic relief, however become less effective over time and do not attenuate the

331 progression of the disease. Unfortunately, the underlying mechanism of neurodegeneration in

332 Parkinson's disease remains poorly understood. In this study, we have demonstrated that ATF4

333 induction in cellular models of PD triggers neuronal cell death through transcriptional activation

334 of known pro-death genes and subsequent apoptotic processes. Specifically, we have

335 determined that (a) ATF4 expression is increased following neuronal injury caused by PD 
336 neurotoxins (MPP+ and 6-OHDA) as well as by $\alpha$-synuclein aggregates produced by preformed $\alpha$ -

337 synuclein fibrils, that (b) ATF4 is transcriptionally active and promotes the expression of the pro-

338 apoptotic target genes Trib3, CHOP, and PUMA, that (c) ATF4-deficient dopaminergic neurons

339 are protected in cellular PD models, and importantly that $(d)$ pharmacological inhibition of ATF4

340 attenuates neuronal loss in PD paradigms.

342 ATF4 is known to induce the expression of genes involved mitigating cellular stresses but during

343 prolonged activation is also known to induce the expression of pro-apoptotic factors (Harding et

344 al., 2003; Zinszner et al., 1998). Therefore, it is not surprising that ATF4 has been reported to

345 have both pro-survival and pro-death effects in neurons. Specifically, ATF4 has been reported to

346 promote resistance of HT22 and PC12 cells to glutamate induced oxytosis by inducing the

347 expression of the cystine/glutamate antiporter- xCT (Lewerenz et al., 2011; Lewerenz \& Maher,

348 2009). Conversely, in another study it was shown that ATF4-deficient cortical neurons are

349 resistant to homocysteine induced oxidative stress and that overexpression of ATF4 is sufficient

350 to promote neuronal death (Lange et al., 2008). Furthermore, we have previously demonstrated

351 that ATF4-deficient neurons are resistant to ER stress but not DNA damage induced apoptosis

352 (Galehdar et al., 2010). In this study we show that ATF4 functions as a pro-death effector in both

353 neurotoxin and pathological $\alpha$-synuclein models of PD. In agreement with our findings that ATF4

354 induction promotes dopaminergic neuronal loss, Gully et al., (2016) demonstrated that

355 overexpression of ATF4 is sufficient to induce dopaminergic neuron loss in the substantia nigra in

356 vivo. In contrast, it has been reported that in PC12 cells ATF4 expression induces Parkin

357 expression and promotes cell survival in PD toxin paradigms (Sun et al., 2013). However, a more 
recent study by this group demonstrated that the ATF4 target Trib3 is induced in cellular models

359 of PD and promotes neuronal death by decreasing Parkin levels in neurons (Aimé et al., 2015).

360 Although our results demonstrate that ATF4 is a key regulator of neuronal death in cellular

361 models of PD, future studies will be needed to evaluate the role of ATF4 in in vivo models of PD.

362 However, this will require the generation of brain specific conditional knockout models as adult

363 ATF4 germline knockout mice exhibit peripheral phenotypes including decreased bone mass and

364 microphthalmia (defect in eye lens formation that renders them essentially blind) and thus are

365 unsuitable for motor or cognitive behavioural testing (Hettmann, Barton, \& Leiden, 2000;

366 Masuoka \& Townes, 2002). Interestingly, ATF4 has been implicated in other neurodegenerative

367 conditions but the mechanisms by which it regulates neuronal loss have yet to be fully

368 understood. Specifically, in Alzheimer's disease, elevated levels of ATF4 has been found in post-

369 mortem patient brains and in mice amyloid-beta causes ATF4 production within axons leading to

370 neurodegeneration (Baleriola et al., 2014). Furthermore, in a mouse model of Amyotrophic

371 Lateral Sclerosis (ALS), ATF4-null transgenic ALS animals exhibited delayed onset of disease and

372 displayed a prolonged lifespan compared ATF4-wild type/ALS animals (Matus, Lopez, Valenzuela,

373 Nassif, \& Hetz, 2013).

375 In this study, we demonstrated that ATF4 is required for the transcriptional activation of known 376 pro-apoptotic factors CHOP, Trib3, and the Bcl-2 family member PUMA. Interestingly, all three of

377 these factors have previously been reported to contribute to dopaminergic neuronal death in PD

378 models (Aimé et al., 2015; Bernstein et al., 2011; Bernstein \& O’Malley, 2013; Silva et al., 2005).

379 However, the mechanism by which these targets lead to neuronal loss is not completely 
understood. CHOP and Trib3 have previously been reported to negatively regulate the

381 serine/threonine survival kinase Akt, leading to dephosphorylation and nuclear translocation of

382 different FoxO transcription factors (Du, Herzig, Kulkarni, \& Montminy, 2003; Ghosh, Klocke,

383 Ballestas, \& Roth, 2012). In an amyloid-beta toxicity study, Saleem and Biswas (2017) described

384 an increase in Trib3 levels in both in vivo and in vitro experiments which leads to downregulation

385 of Akt and initiation of FoxO1 translocation. We have previously demonstrated that trophic

386 factor deprivation downregulates Akt and promotes FoxO3a mediated transcriptional activation

387 of PUMA suggesting that these apoptotic factors may be involved in a common apoptotic

388 pathway (Ambacher et al., 2012). Indeed, we have previously shown PUMA to be a key regulator

389 of oxidative stress and ER-stress induced neuronal apoptosis (Steckley et al., 2007; Galehdar et al

390 2010).

392 The ISR is activated in many neurodegenerative disorders and several studies have investigated

393 the therapeutic potential of targeting elF2 $\alpha$ kinases in mouse models (Hugon, Mouton-Liger,

394 Dumurgier, \& Paquet, 2017; Scheper \& Hoozemans, 2013). Related to PD it has recently been

395 reported that PERK signalling is elevated in PD brains and in the SNpc of rodents treated with 6-

396 OHDA (Mercado et al., 2018b). Furthermore, they demonstrated that administration of the PERK

397 inhibitor GSK2606414 protected nigrostriatal neurons against 6-OHDA induced toxicity and

398 improved motor function. In this study we found that the PKR inhibitor C16 reduced ATF4

399 induction and ATF4 mediated transcriptional induction of the pro-apoptotic targets Chop and

400 Trib3. Furthermore, we found that C16 markedly reduced MPP+ and 6-OHDA induced

401 dopaminergic neuron death in mesencephalic cultures. Interestingly, we found that C16 also 

attenuated ATF4 activation by oxidative stressors and the classic ER stressor thapsigargin. ER

403 stress is generally thought to activate ATF4 via the PERK pathway suggesting that C16 may be

404 able to inhibit PERK in addition to PKR. However, we can also not rule out the possibility that C16

405 may be affecting ATF4 activation via an off-target mechanism. Indeed, it has previously been

406 reported that C16 can also exert neuroprotection via PKR-independent mechanisms possibly

407 involving inhibition of CDK5 (Chen, Wang, \& D'Mello, 2008). Considering this, further studies

408 should aim to better understand the mechanism by which C16 exerts its neuroprotective effects

409 and how this compound may contribute to a reduction in ATF4-induced neuronal cell death. 
ATF4 in PD Neuronal Death

MD Demmings

\section{References}

Aimé, P., Sun, X., Zareen, N., Rao, A., Berman, Z., Volpicelli-Daley, L., ... Greene, L. A. (2015). Trib3

Ambacher, K. K., Pitzul, K. B., Karajgikar, M., Hamilton, A., Ferguson, S. S., \& Cregan, S. P. (2012). Is Elevated in Parkinson's Disease and Mediates Death in Parkinson's Disease Models. The Journal of Neuroscience: The Official Journal of the Society for Neuroscience, 35(30), 10731-10749. https://doi.org/10.1523/JNEUROSCI.0614-15.2015

The JNK- and AKT/GSK3 $\beta$ - Signaling Pathways Converge to Regulate Puma Induction and Neuronal Apoptosis Induced by Trophic Factor Deprivation. PLOS ONE, 7(10). https://doi.org/10.1371/journal.pone.0046885

Baba, M., Nakajo, S., Tu, P. H., Tomita, T., Nakaya, K., Lee, V. M., ... Iwatsubo, T. (1998). Aggregation of alpha-synuclein in Lewy bodies of sporadic Parkinson's disease and dementia with Lewy bodies. The American Journal of Pathology, 152(4), 879-884. Retrieved from http://www.ncbi.nlm.nih.gov/pubmed/9546347

Baleriola, J., Walker, C. A., Jean, Y. Y., Crary, J. F., Troy, C. M., Nagy, P. L., \& Hengst, U. (2014). Axonally Synthesized ATF4 Transmits a Neurodegenerative Signal across Brain Regions. https://doi.org/10.1016/j.cell.2014.07.001

Bernstein, A. I., Garrison, S. P., Zambetti, G. P., \& O'malley, K. L. (2011). 6-OHDA generated ROS induces DNA damage and p53-and PUMA-dependent cell death. https://doi.org/10.1186/1750-1326-6-2

Bernstein, A. I., \& O'Malley, K. L. (2013). MPP+-induces PUMA- and p53-dependent, but ATF3independent cell death. Toxicology Letters, 219(2), 93-98. https://doi.org/10.1016/j.toxlet.2013.03.003

Bosco, D. A., Fowler, D. M., Zhang, Q., Nieva, J., Powers, E. T., Wentworth, P., ... Kelly, J. W. (2006). Elevated levels of oxidized cholesterol metabolites in Lewy body disease brains accelerate a-synuclein fibrilization. Nature Chemical Biology. https://doi.org/10.1038/nchembio782

Bove, J., Prou, D., Perier, C., \& Przedborski, S. (2005). Toxin-Induced Models of Parkinson 's Disease, 2(July), 484-494.

Chen, H.-M., Wang, L., \& D'Mello, S. R. (2008). A chemical compound commonly used to inhibit 
ATF4 in PD Neuronal Death

MD Demmings

PKR, \{8-(imidazol-4-ylmethylene)-6H-azolidino[5,4-g] benzothiazol-7-one\}, protects neurons by inhibiting cyclin-dependent kinase. European Journal of Neuroscience, 28(10), 20032016. https://doi.org/10.1111/j.1460-9568.2008.06491.x

Clark, I. E., Dodson, M. W., Jiang, C., Cao, J. H., Huh, J. R., Seol, J. H., ... Guo, M. (2006). Drosophila pink1 is required for mitochondrial function and interacts genetically with parkin. Nature, 441(7097), 1162-1166. https://doi.org/10.1038/nature04779

Colla, E., Coune, P., Liu, Y., Pletnikova, O., Troncoso, J. C., Iwatsubo, T., ... Lee, M. K. (2012). Endoplasmic reticulum stress is important for the manifestations of $\alpha$-synucleinopathy in vivo. Journal of Neuroscience, 32(10), 3306-3320. https://doi.org/10.1523/JNEUROSCI.5367-11.2012

Cregan, S. P., Fortin, A., MacLaurin, J. G., Callaghan, S. M., Cecconi, F., Yu, S. W., ... Slack, R. S. (2002). Apoptosis-inducing factor is involved in the regulation of caspase-independent neuronal cell death. Journal of Cell Biology, 158(3), 507-517. https://doi.org/10.1083/jcb.200202130

Damier, P., Hirsch, E. C., Agid, Y., \& Graybiel, A. M. (1999). The substantia nigra of the human brain: II. Patterns of loss of dopamine-containing neurons in Parkinson's disease. Brain, 122(8), 1437-1448. https://doi.org/10.1093/brain/122.8.1437

Donnelly, N., Gorman, A. M., Gupta, S., \& Samali, A. (2013). The elF2 a kinases: Their structures and functions. Cellular and Molecular Life Sciences, 70(19), 3493-3511. https://doi.org/10.1007/s00018-012-1252-6

Du, K., Herzig, S., Kulkarni, R. N., \& Montminy, M. (2003). TRB3: A tribbles homolog that inhibits Akt/PKB activation by insulin in liver. Science, 300(5625), 1574-1577. https://doi.org/10.1126/science.1079817

Fallon, J., Matthews, R. T., Hyman, B. T., \& Beal, M. F. (1997). MPP+Produces Progressive Neuronal Degeneration Which Is Mediated by Oxidative Stress. Experimental Neurology, 144(1), 193-198. https://doi.org/10.1006/EXNR.1997.6416

Fawcett, T. W., Martindale, J. L., Guyton, K. Z., Hai, T., \& Holbrook, N. J. (1999). Complexes containing activating transcription factor (ATF)/CAMP-responsive-element-binding protein (CREB) interact with the CCAAT/enhancer-binding protein (C/EBP)-ATF composite site to regulate Gadd153 expression during the stress response. Biochemical Journal, 339(1), 135141. https://doi.org/10.1042/0264-6021:3390135

Fujiwara, H., Hasegawa, M., Dohmae, N., Kawashima, A., Masliah, E., Goldberg, M. S., ... Iwatsubo, T. (2002). a-Synuclein is phosphorylated in synucleinopathy lesions. Nature Cell 
ATF4 in PD Neuronal Death

MD Demmings

$$
\text { Biology, 4(2), 160-164. https://doi.org/10.1038/ncb748 }
$$

Galehdar, Z., Swan, P., Fuerth, B., Callaghan, S. M., Park, D. S., \& Cregan, S. P. (2010). Cellular/Molecular Neuronal Apoptosis Induced by Endoplasmic Reticulum Stress Is Regulated by ATF4-CHOP-Mediated Induction of the Bcl-2 Homology 3-Only Member PUMA. https://doi.org/10.1523/JNEUROSCI.1598-10.2010

Gaven, F., Marin, P., \& Claeysen, S. (2014). Primary Culture of Mouse Dopaminergic Neurons. Journal of Visualized Experiments, (91). https://doi.org/10.3791/51751

Geisler, S., Holmström, K. M., Treis, A., Skujat, D., Weber, S. S., Fiesel, F. C., ... Springer, W. (2010). The PINK1/Parkin-mediated mitophagy is compromised by PD-associated mutations. Autophagy, 6(7), 871-878. https://doi.org/10.4161/auto.6.7.13286

Ghosh, A. P., Klocke, B. J., Ballestas, M. E., \& Roth, K. A. (2012). CHOP Potentially Co-Operates with FOXO3a in Neuronal Cells to Regulate PUMA and BIM Expression in Response to ER Stress CHOP Potentially Co-Operates with FOXO3a in Neuronal Cells to Regulate PUMA and BIM Expression in Response to. PLOS ONE, 7(6), 39586. https://doi.org/10.1371/journal.pone.0039586

Gully, J. C., Sergeyev, V. G., Bhootada, Y., Mendez-Gomez, H., Meyers, C. A., Zolotukhin, S., ... Gorbatyuk, O. S. (2016). Up-regulation of activating transcription factor 4 induces severe loss of dopamine nigral neurons in a rat model of Parkinson's disease. Neuroscience Letters, 627, 36-41. https://doi.org/10.1016/j.neulet.2016.05.039

Harding, H. P., Zhang, Y., Zeng, H., Novoa, I., Lu, P. D., Calfon, M., ... Ron, D. (2003). An integrated stress response regulates amino acid metabolism and resistance to oxidative stress. Molecular Cell, 11(3), 619-633. https://doi.org/10.1016/S1097-2765(03)00105-9

Heman-Ackah, S. M., Manzano, R., Hoozemans, J. J. M., Scheper, W., Flynn, R., Haerty, W., ... Dunn, W. (2017). Alpha-synuclein induces the unfolded protein response in Parkinson's disease SNCA triplication iPSC-derived neurons. Human Molecular Genetics, 26(22), 44414450. https://doi.org/10.1093/hmg/ddx331

Hettmann, T., Barton, K., \& Leiden, J. M. (2000). Microphthalmia due to p53-mediated apoptosis of anterior lens epithelial cells in mice lacking the CREB-2 transcription factor. Developmental Biology. https://doi.org/10.1006/dbio.2000.9699

Holtz, W. A., \& O'Malley, K. L. (2003). Parkinsonian mimetics induce aspects of unfolded protein response in death of dopaminergic neurons. Journal of Biological Chemistry, 278(21), 19367-19377. https://doi.org/10.1074/jbc.M211821200

Hoozemans, J. J. M., van Haastert, E. S., Eikelenboom, P., de Vos, R. A. I., Rozemuller, J. M., \& Scheper, W. (2007). Activation of the unfolded protein response in Parkinson's disease. Biochemical and Biophysical Research Communications, 354(3), 707-711. 
ATF4 in PD Neuronal Death

MD Demmings

https://doi.org/10.1016/j.bbrc.2007.01.043

Hugon, J., Mouton-Liger, F., Dumurgier, J., \& Paquet, C. (2017). PKR involvement in Alzheimer's disease. Alzheimer's Research and Therapy, 9(1), 1-10. https://doi.org/10.1186/s13195017-0308-0

Kaushik, S., \& Cuervo, A. M. (2015). Proteostasis and aging. Nature Medicine, 21(12), 1406-1415. https://doi.org/10.1038/nm.4001

Lange, P., Chavez, J., Pinto, J., Coppola, G., Sun, C., Townes, T., ... Ratan, R. (2008). ATF4 is an oxidative stress - inducible, prodeath transcription factor in neurons in vitro and in vivo. https://doi.org/10.1084/jem.20071460

Lee, J.-Y., Nagano, Y., Taylor, J. P., Lim, K. L., \& Yao, T.-P. (2010). Disease-causing mutations in parkin impair mitochondrial ubiquitination, aggregation, and HDAC6-dependent mitophagy. The Journal of Cell Biology, 189(4), 671-678. https://doi.org/10.1083/jcb.201001039

Lewerenz, J, Sato, H., Albrecht, P., Henke, N., Noack, R., Methner, A., \& Maher, P. (2011). Mutation of ATF4 mediates resistance of neuronal cell lines against oxidative stress by inducing xCT expression. Cell Death and Differentiation, 19, 847-858. https://doi.org/10.1038/cdd.2011.165

Lewerenz, Jan, \& Maher, P. (2009). Basal levels of elF2 $\alpha$ phosphorylation determine cellular antioxidant status by regulating ATF4 and xCT expression. Journal of Biological Chemistry, 284(2), 1106-1115. https://doi.org/10.1074/jbc.M807325200

Lotharius, J., Dugan, L. L., \& O’Malley, K. L. (1999). Distinct mechanisms underlie neurotoxinmediated cell death in cultured dopaminergic neurons. The Journal of Neuroscience: The Official Journal of the Society for Neuroscience, 19(4), 1284-1293. Retrieved from http://www.ncbi.nlm.nih.gov/pubmed/9952406

Lu, P. D., Jousse, C., Marciniak, S. J., Zhang, Y., Novoa, I., Scheuner, D., ... Harding, H. P. (2004). Cytoprotection by pre-emptive conditional phosphorylation of translation initiation factor 2. EMBO Journal, 23(1), 169-179. https://doi.org/10.1038/sj.emboj.7600030

Masuoka, H. C., \& Townes, T. M. (2002). Targeted disruption of the activating transcription factor 4 gene results in severe fetal anemia in mice. Retrieved from www.bloodjournal.org

Matus, S., Lopez, E., Valenzuela, V., Nassif, M., \& Hetz, C. (2013). Functional Contribution of the Transcription Factor ATF4 to the Pathogenesis of Amyotrophic Lateral Sclerosis. PLOS ONE, 8(7), 1-12. https://doi.org/10.1371/journal.pone.0066672

Mercado, G., Castillo, V., Soto, P., López, N., Axten, J. M., Sardi, S. P., ... Hetz, C. (2018a). Targeting PERK signaling with the small molecule GSK2606414 prevents neurodegeneration 
ATF4 in PD Neuronal Death

MD Demmings

in a model of Parkinson's disease. Neurobiology of Disease, 112, 136-148. https://doi.org/10.1016/j.nbd.2018.01.004

Mercado, G., Castillo, V., Soto, P., López, N., Axten, J. M., Sardi, S. P., ... Hetz, C. (2018b). Targeting PERK signaling with the small molecule GSK2606414 prevents neurodegeneration in a model of Parkinson's disease. Neurobiology of Disease, 112(January), 136-148. https://doi.org/10.1016/j.nbd.2018.01.004

Mercado, G., Castillo, V., Soto, P., \& Sidhu, A. (2016). ER stress and Parkinson's disease: Pathological inputs that converge into the secretory pathway. Brain Research, 1648, 626632. https://doi.org/10.1016/j.brainres.2016.04.042

Michel, P. P., Hirsch, E. C., \& Hunot, S. (2016). Understanding Dopaminergic Cell Death Pathways in Parkinson Disease. Neuron, 90(4), 675-691. https://doi.org/10.1016/j.neuron.2016.03.038

Nagatsu, T., Levitt, M., \& Udenfriend, S. (1964). The initial step. Journal of Biological Chemistry, 239(September), 2910-2917. https://doi.org/10.1097/00005792-196405000-00019

Narendra, D. P., Jin, S. M., Tanaka, A., Suen, D. F., Gautier, C. A., Shen, J., ... Youle, R. J. (2010). PINK1 is selectively stabilized on impaired mitochondria to activate Parkin. PLoS Biology, 8(1). https://doi.org/10.1371/journal.pbio.1000298

Ohoka, N., Yoshii, S., Hattori, T., Onozaki, K., \& Hayashi, H. (2005). TRB3, a novel ER stressinducible gene, is induced via ATF4-CHOP pathway and is involved in cell death. EMBO Journal, 24(6), 1243-1255. https://doi.org/10.1038/sj.emboj.7600596

Pakos-Zebrucka, K., Koryga, I., Mnich, K., Ljujic, M., Samali, A., \& Gorman, A. M. (2016). The integrated stress response. EMBO Reports, 17(10), 1374-1395. https://doi.org/10.15252/embr.201642195

Postuma, R. B., Berg, D., Stern, M., Poewe, W., Marek, K., \& Litvan, I. (2015). MDS Clinical Diagnostic Criteria for Parkinson' s Disease. Movement Disorders, 30(12), 1591-1599. https://doi.org/10.1002/mds.26424

Przedborski, S., \& Ischiropoulos, H. (2005). Reactive Oxygen and Nitrogen Species: Weapons of Neuronal Destruction in Models of Parkinson's Disease. Antioxidants \& Redox Signaling, 7(5-6), 685-693. https://doi.org/10.1089/ars.2005.7.685

Ryu, E. J., Harding, H. P., Angelastro, J. M., Vitolo, O. V., Ron, D., \& Greene, L. A. (2002). Endoplasmic reticulum stress and the unfolded protein response in cellular models of 
ATF4 in PD Neuronal Death

MD Demmings

Parkinson's disease. Journal of Neuroscience, 22(24), 10690-10698.

Saleem, S., \& Biswas, S. C. (2017). Tribbles Pseudokinase 3 Induces Both Apoptosis and Autophagy in Amyloid- $\beta$-induced Neuronal Death. Journal of Biological Chemistry, 292(7), 2571-2585. https://doi.org/10.1074/jbc.M116.744730

Scheper, W., \& Hoozemans, J. J. M. (2013). A new PERKspective on neurodegeneration. Science Trans/ational Medicine, 5(206), 3-6. https://doi.org/10.1126/scitranslmed.3007641

Silva, R. M., Ries, V., Oo, T. F., Yarygina, O., Jackson-Lewis, V., Ryu, E. J., ... Burke, R. E. (2005). CHOP/GADD153 is a mediator of apoptotic death in substantia nigra dopamine neurons in an in vivo neurotoxin model of parkinsonism. Journal of Neurochemistry, 95(4), 974-986. https://doi.org/10.1111/j.1471-4159.2005.03428.x

Singleton, D. C., \& Harris, A. L. (2012). Targeting the ATF4 pathway in cancer therapy. Expert Opinion on Therapeutic Targets, 16(12), 1189-1202. https://doi.org/10.1517/14728222.2012.728207

Slodzinski, H., Moran, L. B., Michael, G. J., Wang, B., Novoselov, S., Cheetham, M. E., ... Graeber, M. B. (2009). Homocysteine-induced endoplasmic reticulum protein (herp) is up-regulated in parkinsonian substantia nigra and present in the core of Lewy bodies. Clinical Neuropathology, 28(5), 333-343. Retrieved from http://www.ncbi.nlm.nih.gov/pubmed/19788048

Spillantini, G. M., Schmidt, M. L., Lee, V. M.-Y., Trojanowski, J. Q., Jakes, R., \& Goedert, M. (1997). a-Synuclein in Lewy bodies. Nature, 388, 839-840. https://doi.org/10.1038/42166

Steckley, D., Karajgikar, M., Dale, L. B., Fuerth, B., Swan, P., Drummond-Main, C., ... Cregan, S. P. (2007). Puma Is a Dominant Regulator of Oxidative Stress Induced Bax Activation and Neuronal Apoptosis. Journal of Neuroscience, 27(47), 12989-12999. https://doi.org/10.1523/jneurosci.3400-07.2007

Sun, X., Liu, J., Crary, J. F., Malagelada, C., Sulzer, D., Greene, L. A., \& Levy, O. A. (2013). Neurobiology of Disease ATF4 Protects Against Neuronal Death in Cellular Parkinson's Disease Models by Maintaining Levels of Parkin. https://doi.org/10.1523/JNEUROSCI.229212.2013

Volpicelli-Daley, L. A., Luk, K. C., \& Lee, V. M. Y. (2014). Addition of exogenous $\alpha$-synuclein preformed fibrils to primary neuronal cultures to seed recruitment of endogenous $\alpha$ synuclein to Lewy body and Lewy neurite-like aggregates. Nature Protocols, 9(9), 21352146. https://doi.org/10.1038/nprot.2014.143

Volpicelli-Daley, L. A., Luk, K. C., Patel, T. P., Tanik, S. A., Riddle, D. M., Stieber, A., ... Lee, V. M.-Y. (2011). Exogenous $\alpha$-synuclein fibrils induce Lewy body pathology leading to synaptic 
ATF4 in PD Neuronal Death

MD Demmings

686

687

688

689

690

691

692

693

694

695

696

697

698

699

700

701

702

703

704

705

706

707

708

709

710

711

712

713

714

715

716

717
Zeng, X.-S., Geng, W.-S., Jia, J.-J., Chen, L., \& Zhang, P.-P. (2018). Cellular and Molecular Basis of Neurodegeneration in Parkinson Disease. Frontiers in Aging Neuroscience, 10(109), 1-16. https://doi.org/10.3389/fnagi.2018.00109

Zinszner, H., Kuroda, M., Wang, X. Z., Batchvarova, N., Lightfoot, R. T., Remotti, H., ... Ron, D. (1998). CHOP is implicated in programmed cell death in response to impaired function of the endoplasmic reticulum. Genes and Development, 12(7), 982-995.

https://doi.org/10.1101/gad.12.7.982 
A
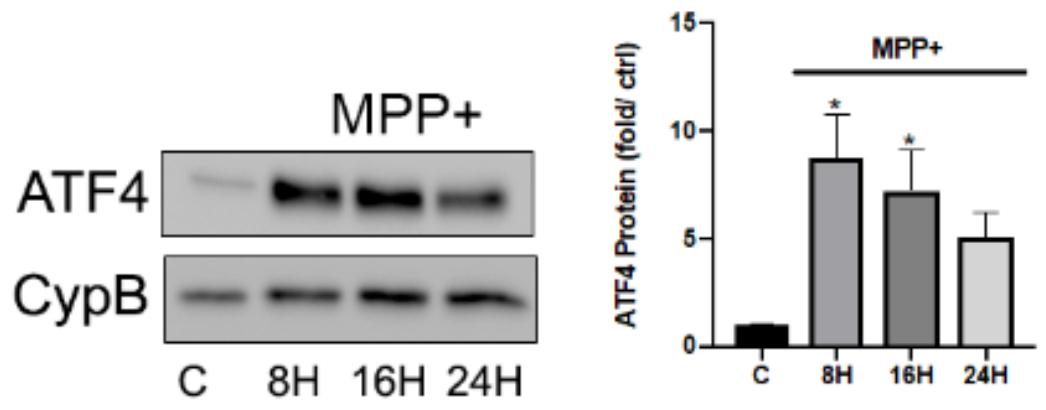

B
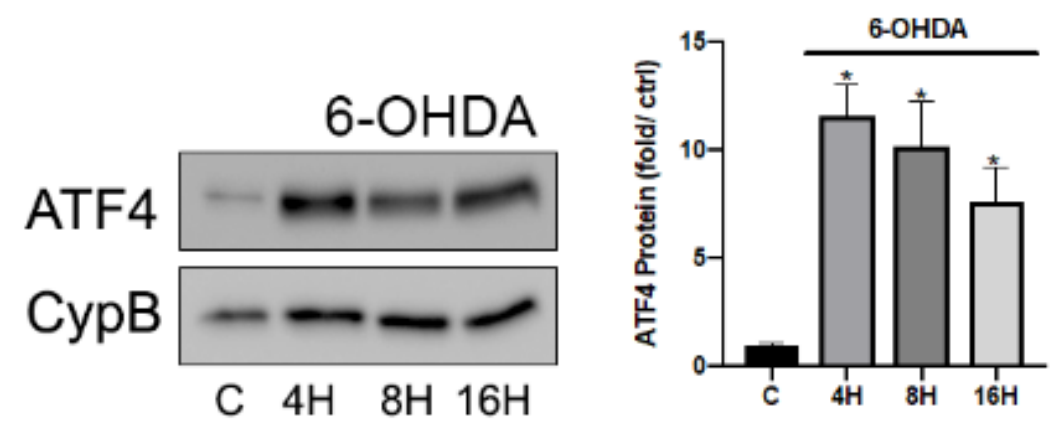

718

Figure 1. PD neurotoxins $M P P+$ and 6-OHDA cause sustained induction of ATF4 in neurons. Cortical neurons were treated with MPP+ [50 $\mu \mathrm{M}](\mathrm{A})$ or 6-OHDA [10 $\mu \mathrm{M}](\mathrm{B})$ and at the indicated timepoints ATF4 protein levels were assessed by Western blot analysis and quantified by densitometry $(n=3 ; * p<0.05)$. C, Cortical neurons were treated with MPP+ $[50 \mu \mathrm{M}]$ or $6-$ OHDA $[10 \mu \mathrm{M}]$ for $8 \mathrm{~h}$ and mRNA levels of ATF4 was determined by quantitative RT-PCR. Induction was normalized to S12 mRNA levels and is reported as fold increase over untreated neurons $(n=3 ; p>0.05)$ 

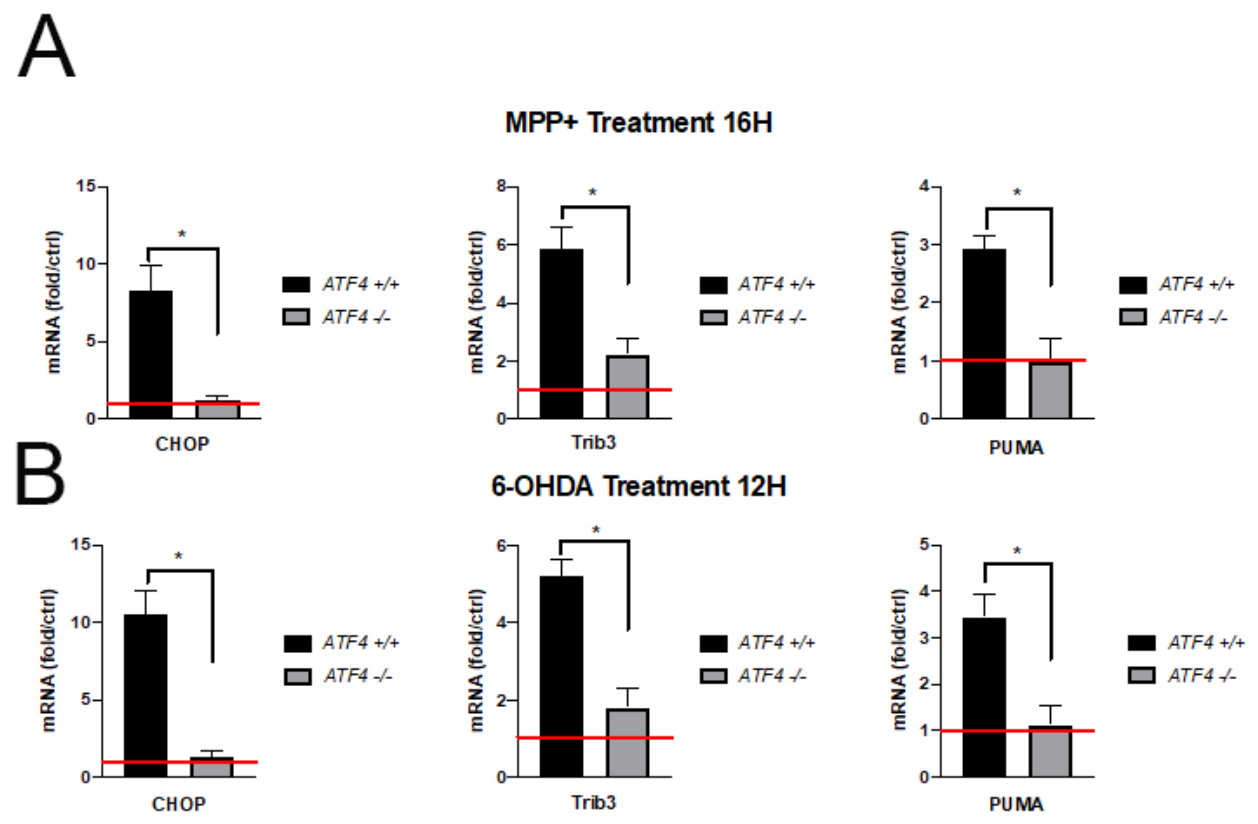

Figure 2. PD mimetics trigger ATF4-dependent transcription of the pro-apoptotic genes Chop, Trb3 and Puma. Cortical neurons derived from ATF4-wildtype and ATF4-null littermates were treated with (A) MPP+ [50 $\mu \mathrm{M}]$ for $16 \mathrm{~h}$ or (B) 6-OHDA [10 $\mu \mathrm{M}]$ for $12 \mathrm{~h}$ and mRNA levels of indicated transcripts were determined by quantitative RT-PCR. Induction was normalized to S12 mRNA levels and is reported as fold increase over untreated neurons $\left(n=4 ;{ }^{*}<0.05\right)$.

741 

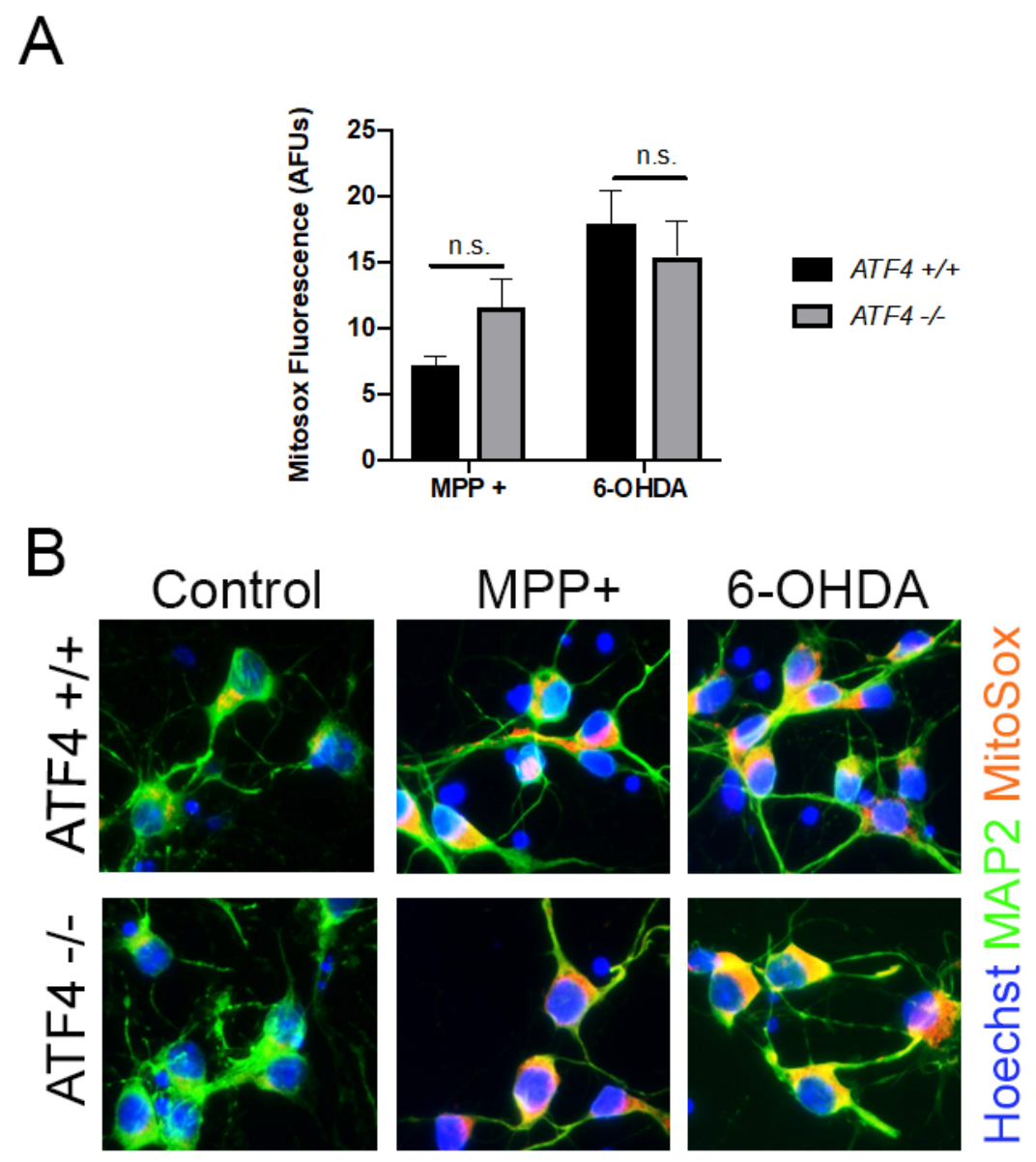

Figure 3. Oxidative stress produced by MPP+ and 6-OHDA is not dependent on ATF4 expression. B, Cortical neurons were treated with MPP $+[50 \mu \mathrm{M}]$ or $6-\mathrm{OHDA}[10 \mu \mathrm{M}]$ and mitochondrial superoxide accumulation was measured and quantified at $8 \mathrm{~h}(\mathrm{n}=3 ; \mathrm{p}>0.05)$. $\mathrm{B}$, Representative images of MAP2-positve (green) neuronal cultures and accumulated superoxide as indicative by MitoSox (red) fluorescence. 

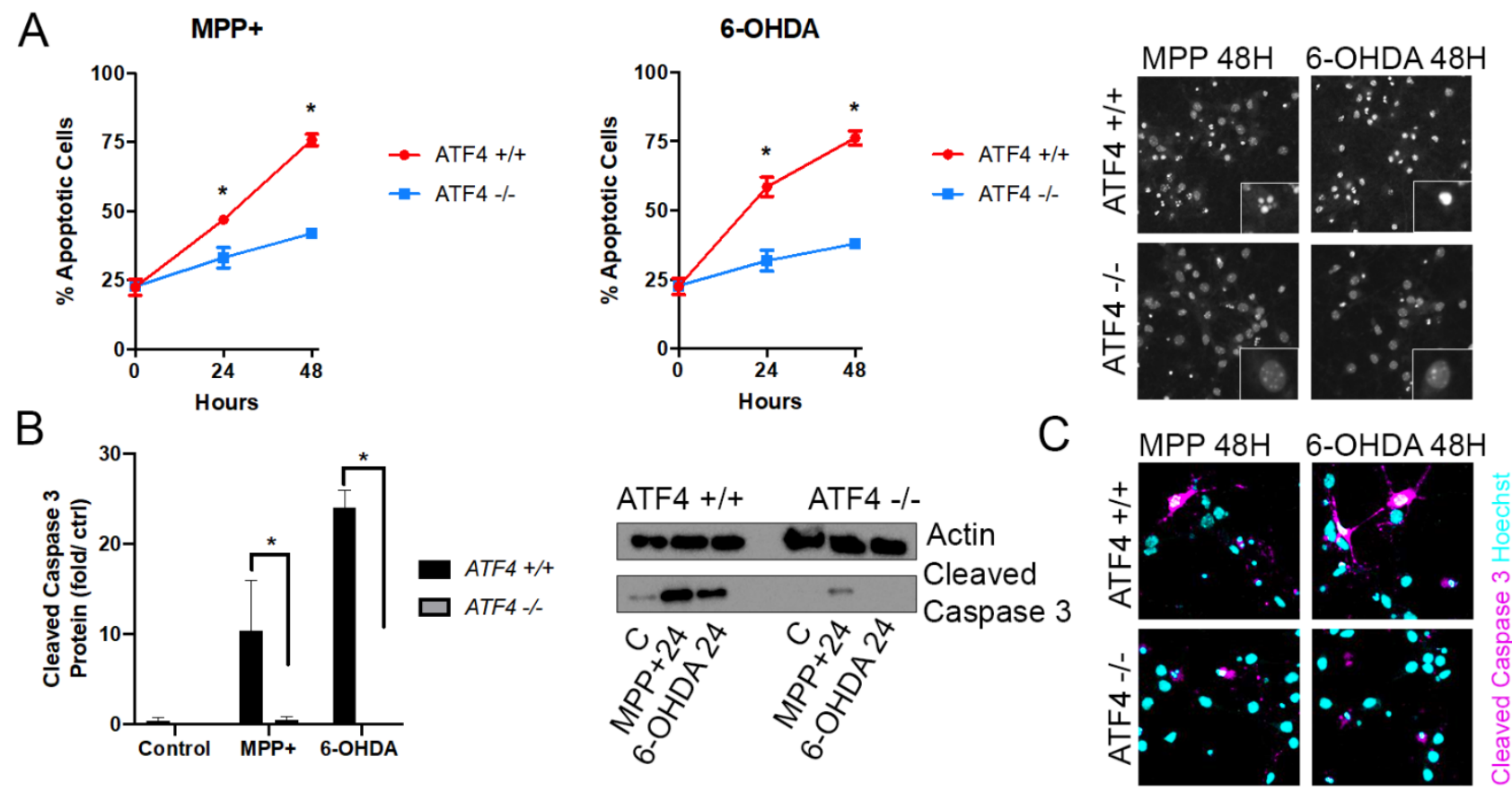

$\mathrm{D}$
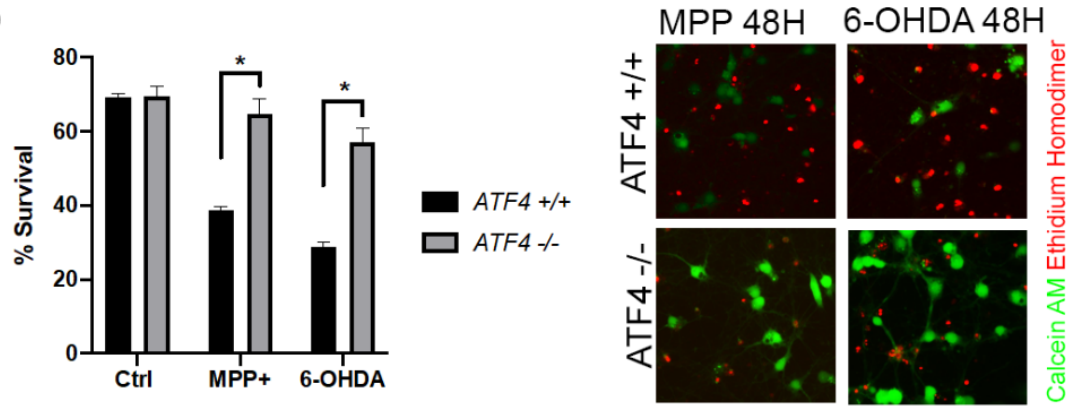

752

753

754

755

756

757

758

759

760

761

762

763

764

765

766

767

768
Figure 4. ATF4 is required for PD toxin-induced neuronal apoptosis. A, cortical neurons derived from ATF4-wildtype and ATF4-null littermates were treated with MPP+ $[50 \mu \mathrm{M}]$ or 6OHDA $[10 \mu \mathrm{M}]$ and the percentage of apoptotic cells was determined by Hoechst 33442 staining at 24 and 48h. $\left(\mathrm{n} \geq 5 ;{ }^{*} \mathrm{p}<0.05\right)$. Representative images of Hoechst 33442 stained neurons captured at $48 \mathrm{~h}$. B, cortical neurons derived from ATF4 wild-type and ATF4-null littermates were treated with MPP+ [50 $\mu \mathrm{M}]$ or 6-OHDA $[10 \mu \mathrm{M}]$ and levels of cleaved caspase 3 were assessed at $24 \mathrm{H}$ by Western blot analysis and quantified by densitometry $(n=3 ; * p<0.05)$. C, Representative immunofluorescent images of cleaved caspase 3 in ATF4-wildtype and ATF4null neuronal cultures treated with MPP+ $(50 \mu \mathrm{M})$ or $6-O H D A(10 \mu \mathrm{M})$ for $24 \mathrm{~h}$. D, cortical neurons derived from ATF4-wildtype and ATF4-null littermates were treated with MPP+ [50 $\mu \mathrm{M}]$ and 6-OHDA $[10 \mu \mathrm{M}]$ and survival was assessed by Calcein-AM/ Ethidium-Homodimer (live/dead) staining $\left(\mathrm{n} \geq 4 ;{ }^{*} \mathrm{p}<0.05\right)$. Representative fluorescent images of MPP+ and 6-OHDA treated ATF4-wildtype and ATF4-deficient neurons at 48H using live (green)/dead (red) assay. 


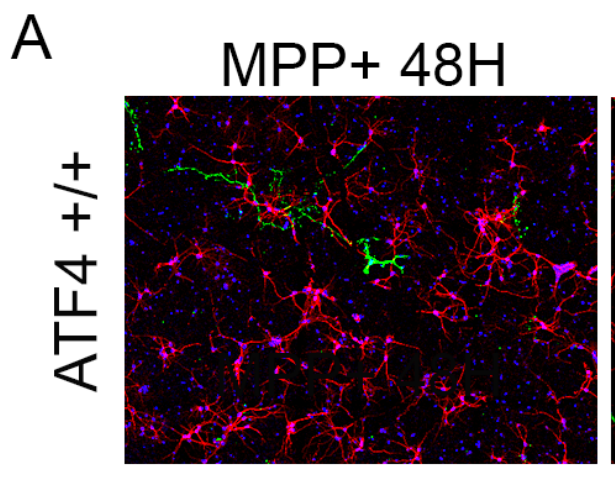

6-OHDA 48H
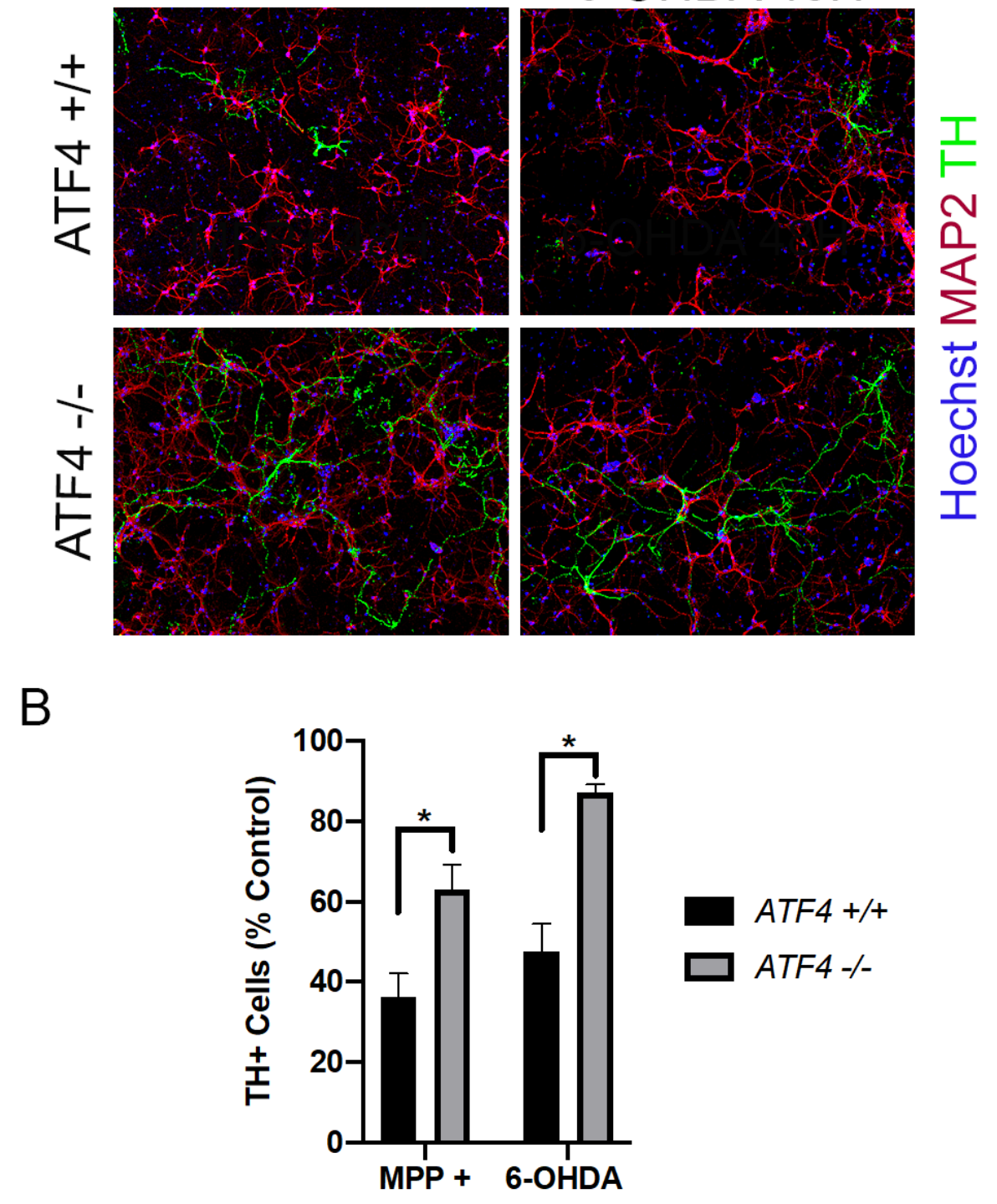

Figure 5. Dopaminergic neurons are conserved in ATF4-null mesencephalic cultures exposed to $M P P+$ or 6-OHDA. Mesencephalic neurons derived from ATF4-wildtype and ATF4-null littermates were treated with MPP+ $[25 \mu \mathrm{M}]$ or $6-\mathrm{OHDA}[5 \mu \mathrm{M}]$ for $48 \mathrm{~h}$ and then immunostained for the dopaminergic neuron marker tyrosine hydroxylase $(\mathrm{TH})$. A, Representative images showing increased numbers of residual dopaminergic neurons $(\mathrm{TH}+$, green) in ATF4-/- mesencephalic cultures compared to ATF4+/+ cultures following treatment wih MPP+ or 6-OHDA. Cultures were co-stained for the general neuronal marker MAP2 and the nuclear stain Hoechst. B, Quantification of the total number of $\mathrm{TH}+$ neurons in ATF4+/+ and ATF4-/- mesencephalic cultures treated with MPP+ $(25 \mu \mathrm{M})$ or $6-\mathrm{OHDA}(5 \mu \mathrm{M})$ for $48 \mathrm{~h}$. TH+ cell counts are reported as a percentage of untreated neurons from the same culture $(n=3$;

$780 * \mathrm{p}<0.05)$. 
A

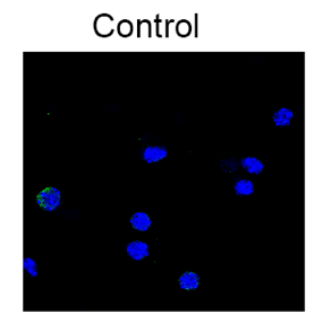

C

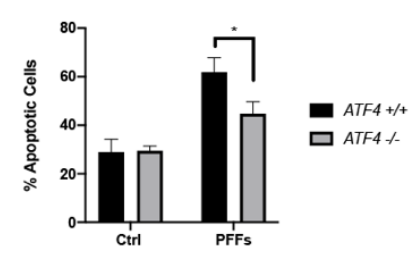

a-syn PFFS

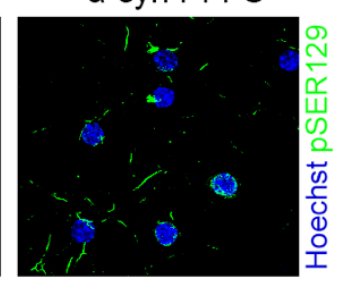

D

B

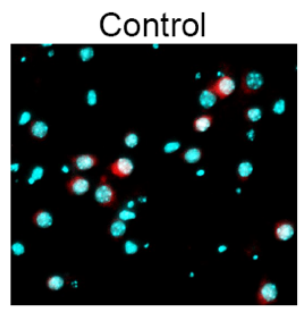
a-syn PFFS
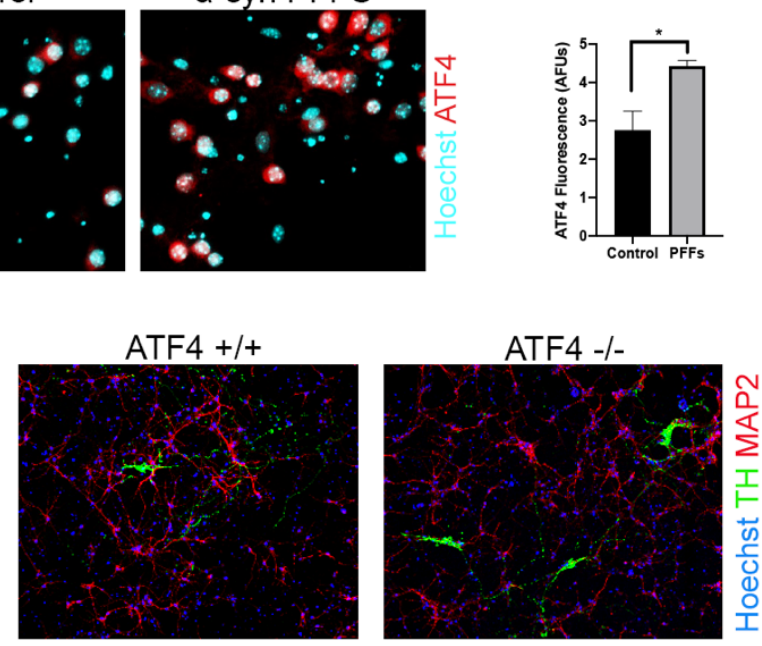

a-syn PFFS

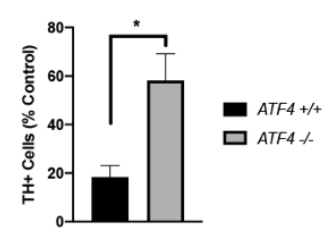

Figure 6. Human alpha-synuclein pre-formed fibrils ( $\alpha$-Syn PFFs) induce ATF4-dependent neuronal death. Cortical neurons were treated with $\alpha$-Syn PFFs $[5 \mu \mathrm{g} / \mathrm{ml}]$ or left untreated (control) for 12 days. A, Representative images showing pathogenic intracellular aggregates of $\alpha$-Synuclein identified by p-Serine129- $\alpha$-synuclein immunoreactivity (green). B, Representative images of ATF4 immunostaining (red) and quantification of ATF4 immunofluorescence intensity $(\mathrm{n}=3 ; * \mathrm{p}<0.05)$. C, Cortical neurons derived from ATF4-wildtype and ATF4-null littermates were treated with $\alpha$-Syn PFFs $[5 \mu \mathrm{g} / \mathrm{ml}]$ for 14 days and the percentage of apoptotic cells was determined by Hoechst staining $(n=4 ; * \mathrm{p}<0.05)$. D, mesencephalic neurons derived from ATF4-wildtype and ATF4-null littermates were treated with PFFs [5 $\mu \mathrm{g} / \mathrm{ml}]$ for 14 days $\left(\mathrm{n}=3 ;{ }^{*}<0.05\right)$. Representative images of dopaminergic $(\mathrm{TH}+)$ neurons (green) in wildtype and ATF4-deficient mesencephalic cultures exposed to $\alpha$-Syn PFFs for 14 days. 

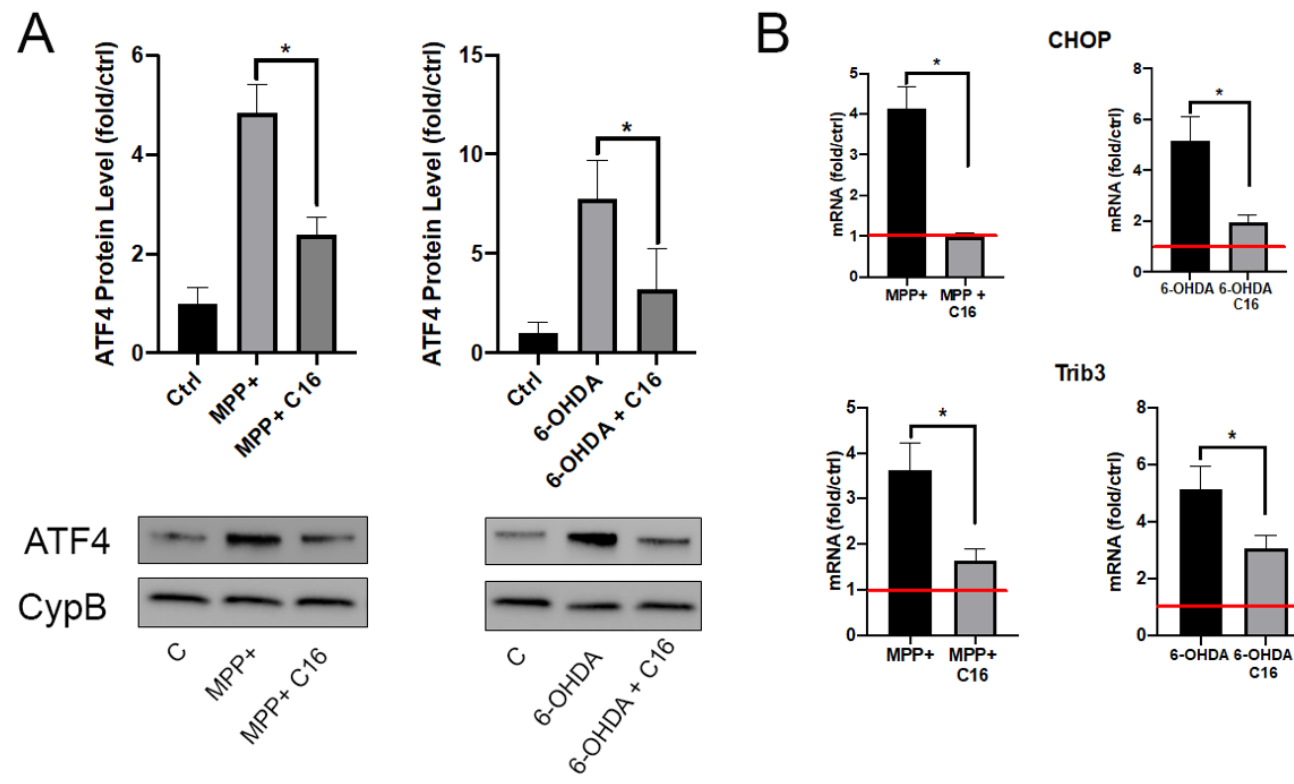

Trib3

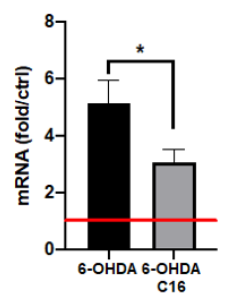

801

C

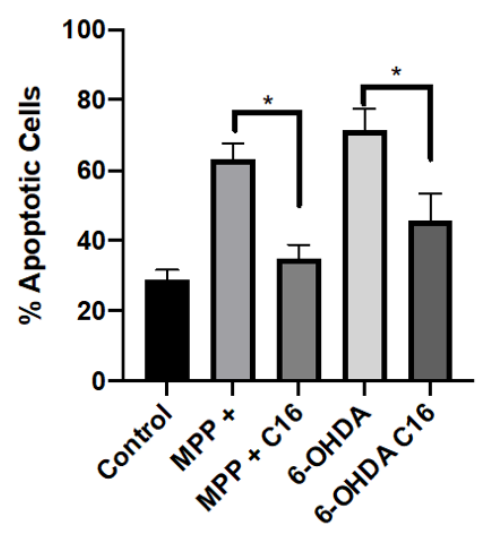

Figure 7. The eIF2a kinase inhibitor C16 attenuates PD neurotoxin induced ATF4 activation and neuronal cell death. A, cortical neurons were treated with MPP+ [50 $\mu \mathrm{M}]$ or 6-OHDA [10 $\mu \mathrm{M}]$ for $8 \mathrm{~h}$ in the presence or absence of $\mathrm{C} 16[2 \mu \mathrm{M}]$ and ATF4 protein levels were assessed by Western blot analysis and quantified by densitometry. ATF4 expression was normalized to Cyclophilin B levels $(\mathrm{n}=3$; * $\mathrm{p}<0.05)$. B, mRNA levels of ATF4-dependent pro-apoptotic targets Chop and Trib3 were determined by quantitative RT-PCR $8 \mathrm{~h}$ following treatment with MPP+ or 6-OHDA in the presence or absence of C16. Induction was normalized to S12 mRNA levels and is reported as fold increase over untreated neurons $\left(n=5 ;{ }^{*}<0.05\right)$. C, cortical neurons were treated with MPP $+[50 \mu \mathrm{M}]$ or $6-\mathrm{OHDA}[10 \mu \mathrm{M}]$ in the presence or absence of $\mathrm{C} 16[2 \mu \mathrm{M}]$ and the percentage of apoptotic cells was determined at 24 hours by Hoechst staining $\left(n=3 ;{ }^{*}<0.05\right)$. $\mathrm{D}$, mRNA levels of UPR marker BiP in cortical neurons were determined by quantitative RTPCR at $8 \mathrm{H}\left(\mathrm{n}=3 ;{ }^{\mathrm{p}}<0.05\right)$ and spliced XBP1 patterns at $8 \mathrm{~h}$ were assessed in triplicate via $\mathrm{qPCR}$ (representative image shown). 